\title{
Can mean platelet volume and neutrophil- to-lymphocyte ratio be biomarkers of acute exacerbation of bronchiectasis in children?
}

\author{
HIKMET T. NACAROGLU ${ }^{1}$, SEMIHA BAHCECI ERDEM ${ }^{1}$, SAIT KARAMAN ${ }^{l}$, SELCUK YAZICI ${ }^{2}$ \\ DEMET CAN ${ }^{I}$
}

${ }^{1}$ Department of Pediatric Allergy and Immunology, Dr. Behcet Uz Children Disease and Surgery Training and Research Hospital, Izmir, Turkey

${ }^{2}$ Department of Paediatrics, Balıkesir University Medical Faculty, Balıkesir, Turkey

\begin{abstract}
Introduction: Bronchiectasis $(B E)$ is a parenchymal lung disease evolving as a result of recurrent lung infections and chronic inflammation. Although it has been shown in adult studies that mean platelet volume (MPV) and neutrophil-to-lymphocyte ratio (NLR) can be used as biomarkers of airway inflammation, knowledge is limited in the paediatric age group. The aim of our study is to investigate the potential of MPV and NLR as biomarkers that may indicate acute exacerbations of non-cystic fibrosis $B E$ in children.

Material and methods: Children with non-cystic fibrosis BE $(n=50)$, who were followed in the division of Paediatric Pulmonology of our hospital between June 2010 and July 2015, were involved in the present retrospective cross-sectional study. Haemogram values during acute exacerbations and non-exacerbation periods, and a control group were compared.

Results: In children with bronchiectasis, the average leukocyte count $(p<0.001)$, platelet count $(p=0.018)$, absolute neutrophil count $(p<0.001)$, and NLR $(p<0.001)$ were higher, as expected, when compared with the control group. NLR values, in the period of acute exacerbation were significantly higher than the values of both the non-exacerbation periods $(p=0.02)$ and the control group $(p<0.001)$. In contrast, MPV values in the period of acute exacerbation did not exhibit a significant difference from those of non-exacerbation periods $(p=0.530)$ and the control group $(p=0.103)$.

Conclusions: It was concluded that leukocyte count, platelet count, absolute neutrophil count, and $N L R$ can be used to show chronic inflammation in BE, but only NLR and absolute neutrophil count can be used as biomarkers to show acute exacerbations.
\end{abstract}

Key words: mean platelet volume, neutrophil-to-lymphocyte ratio, childhood, bronchiectasis.

(Centr Eur J Immunol 2017; 42 (4): 358-362)

\section{Introduction}

Bronchiectasis (BE) is a chronic inflammatory lung disease that leads to persistent productive cough, recurrent lower respiratory tract infections, and obstructive lung disease in children and adults [1]. The disease is characterised by abnormal and persistent dilatation of the airways, which occurs as a result of the chronic inflammation due to insufficient mucosal clearance and recurrent/chronic infections of the airways [2]. The exacerbations observed in BE patients, reduce lung function by increasing the damage in the lung parenchyma, impair quality of life and lead to the development of respiratory failure in patients [3]. In BE patients, identification of biomarkers of inflammation showing relapse is of great importance. Most of the present biomarkers require cooperation of the patient or tend to be invasive, therefore they cannot be used effectively. For example, the spirometric measurements that are often used require effective cooperation and thus have limited use in small children, and methods such as bronchial biopsy and bronchoalveolar lavage (BAL) are not practical because they tend to be invasive $[4,5]$.

Neutrophils and platelets are known to be engaged in the inflammatory process in $\mathrm{BE}$ and to play a role in progressive lung damage [1-3]. Mean platelet volume (MPV) is a value that indicates platelet activation, and can be used as a biomarker in inflammation [6-8]. Also in adult studies, it has been shown that the neutrophil-to-lymphocyte ratio (NLR) may be a biomarker of airway inflammation [9].

Correspondence: Hikmet T. Nacaroglu, Department of Pediatric Allergy and Immunology, Dr. Behcet Uz Children Disease and Surgery Training and Research Hospital, Alsancak/Izmir, 35220 Izmir, Turkey, e-mail: tekin212@gmail.com Submitted: 5.08.2016; Accepted: 12.12.2016 
The aim of our study is to investigate the potential of MPV and NLR as biomarkers that may indicate acute exacerbations of non-cystic fibrosis (CF) BE in children.

\section{Material and methods}

\section{Patient population}

All patients who had a diagnosis of non-CF BE or were hospitalised due to acute exacerbation between June 2010 and July 2015, were included in the present retrospective cross-sectional study. Average age of the 50 children who were hospitalised due to acute exacerbation was detected as $151( \pm 53 \mathrm{SD})$ months. Twenty-four $(48 \%)$ were male and $26(52 \%)$ were female. In the control group, $45(51.7 \%)$ were male, $42(48.3 \%)$ were female, and the average age was 150 ( $\pm 36 \mathrm{SD})$ months. The diagnosis was confirmed by thorax High-Resolution Computed Tomography (HRCT) in the patients who had a persistent wet cough and abnormal chest X-ray findings that did not respond to antibiotic therapy and also maintained non-regressive symptoms for 2-4 weeks [10]. The aetiology of BE was detected as; primary immune deficiency $26 \%$, protracted bacterial bronchitis $22 \%$, bronchiolitis obliterans $18 \%$, primary ciliary dyskinesia $10 \%$, lung disease secondary to gastro-oesophageal reflux $8 \%$, foreign body aspiration $6 \%$, tuberculosis $6 \%$, and congenital malformation $4 \%$. Exacerbation criteria were defined as having more than two of the following signs and symptoms: increase in cough frequency, sputum production with changes in sputum content, dyspnoea, tachypnoea, reduced exercise capacity, loss of appetite, haemoptysis, fever above $38^{\circ} \mathrm{C}$, and clinically determined crackles and wheezing [10]. Children who were diagnosed with sepsis, iron deficiency anaemia, obesity, hyperlipidaemia, diabetes mellitus, hypertension, chronic renal failure, nephrotic syndrome, inflammatory bowel disease, connective tissue disease, or receiving systemic steroids within the last month were excluded from our study because it was previously reported that these diseases affected MPV and NLR values. The demographic parameters of patients and haemogram findings in non-exacerbation periods were obtained from the file records. When patients were hospitalised due to acute exacerbation, full blood count and C-reactive protein (CRP) were studied. Haemogram parameters and CRP values in acute exacerbations (Group 1) and non-exacerbation periods (Group 2) were compared. Eighty-seven healthy children with equivalent age and gender were randomised as the control group. Then the same parameters were compared with the data of the patients in the healthy control group (Group 3). Approval by the local Ethics Committee was granted.

\section{Counting blood samples}

Whole blood count was performed via Beckman Coulter LH 780, and blood samples which were anticoagulated with K3EDTA were used. The Coulter principle is volumetric analysis. Haemoglobin level, white blood cell count (WBC), platelet count, and MPV values were recorded for each patient. The reference range for MPV was between 7.0 and $11.0 \mathrm{fL}$. The neutrophil-lymphocyte ratio was calculated by dividing the percentage of neutrophils and lymphocytes in complete blood count analysis. Serum CRP level was measured using an Abbott c8000 Architect (USA) device.

\section{Statistical analyses}

Statistical Package for Social Sciences (SPSS for Windows 15.0 Chicago, USA) software was used to analyse the data. Results were given as either mean \pm standard deviation (SD) or median (min-max) according to the distribution. The Chi-squared test was used to compare grouped data. The paired $t$-test was used to compare group-specific measurements, and the independent Student's $t$-test to compare measurements among independent groups. Kruskal-Wallis test and Mann-Whitney $U$ tests were used for non-normally distributed variables. Pearson's correlation analysis was

Table 1. Comparison of haemogram parameters and C-reactive protein levels between acute exacerbation (Group 1), non-exacerbation (Group 2), and controls (Group 3)

\begin{tabular}{|c|c|c|c|c|c|c|}
\hline Parameter & $\begin{array}{l}\text { Group } 1 \\
(n=\mathbf{5 0})\end{array}$ & $\begin{array}{c}\text { Group } 2 \\
(n=50)\end{array}$ & $\begin{array}{l}\text { Group } 3 \\
(n=87)\end{array}$ & $1-2 p$ & $2-3 p$ & $1-3 p$ \\
\hline $\mathrm{WBC}\left(\times 10^{3} / \mu \mathrm{l}\right)$ & $10.8 \pm 4.1$ & $8.5 \pm 3.5$ & $7.3 \pm 1.4$ & 0.83 & $<0.001$ & $<0.001$ \\
\hline Absolute neutrophil count $\left(\times 10^{3} / \mu \mathrm{l}\right)$ & $7.3 \pm 4.1$ & $4.9 \pm 3.1$ & $3.8 \pm 1.1$ & 0.001 & $<0.001$ & $<0.001$ \\
\hline Absolute lymphocyte count $\left(\times 10^{3} / \mu \mathrm{l}\right)$ & $2.1 \pm 0.8$ & $2.4 \pm 0.9$ & $2.7 \pm 0.6$ & 0.71 & 0.607 & $<0.001$ \\
\hline NLR & $5.1 \pm 6.3$ & $2.3 \pm 2.2$ & $1.5 \pm 1.2$ & 0.02 & 0.01 & $<0.001$ \\
\hline $\mathrm{CRP}(\mathrm{mg} / \mathrm{l})$ & $4.1 \pm 7.0$ & $0.45 \pm 0.5$ & & $<0.001$ & - & - \\
\hline $\operatorname{Plt}\left(\times 10^{3} / \mu \mathrm{l}\right)$ & $319 \pm 103$ & $336 \pm 106$ & $285 \pm 62$ & 0.284 & 0.001 & 0.018 \\
\hline MPV (fl) & $8.5 \pm 0.9$ & $8.6 \pm 1.09$ & $8.2 \pm 0.7$ & 0.530 & 0.03 & 0.103 \\
\hline
\end{tabular}


used to explore correlations between the measurements. $P$-values $<0.05$ were considered statistically significant.

\section{Results}

Leukocyte count, platelet count, MPV, neutrophil count, absolute lymphocyte count, and NLR values of patients during acute exacerbations and non-exacerbation periods and of control group are shown in Table 1. Platelet counts of both acute exacerbations and non-exacerbation periods were significantly higher than those in the control group ( $p=0.001, p=0.018$ ). Average MPV values of non-exacerbation periods were significantly higher than in the control group $(p=0.03)$. In contrast, the MPV values of acute exacerbation were not statistically different when compared to non-exacerbation periods or the control group ( $p=0.103, p=0.530$, respectively) (Table 1$)$.

Absolute neutrophil counts in the periods of acute exacerbations and non-exacerbation were significantly higher than in the control group $(p<0.001)$. When periods of acute exacerbations and non-exacerbation were compared, absolute neutrophil count was found to be significantly higher in the period of exacerbation $(p=0.001)$ (Table1). NLR revealed a statistically significant increase both in the period of acute exacerbation and during non-exacerbation when compared with the control group $(p=0.02$, $p=0.01)$. Also, when compared to each other, NLR in the exacerbation period was significantly higher than during the period of non-exacerbation $(p<0.001)$ (Table 1). The CRP values in the acute exacerbation period were significantly higher than in the non-exacerbation period $(p<0.001)$ (Table 1). We investigated the correlation between leukocyte count and CRP values with the MPV. When acute exacerbations and non- exacerbation periods were compared, there was no correlation. A moderately $(r=0.498)$ significant, positive correlation was found between NLR and acute exacerbation period CRP levels $(p<0.001)$. Similarly, a moderately $(r=0.663)$ significant positive correlation was found between NLR and acute exacerbation-period leucocyte numbers $(p<0.001)$ (Table 2).

\section{Discussion}

In the present study, which evaluates the potential of haemogram parameters of children with BE (especially MPV and NLR) as biomarkers of acute exacerbation, it was detected that 1) leukocyte count, platelet count, absolute neutrophil count, and NLR show chronic inflammation of BE, and 2) only absolute neutrophil count and NLR can be used as biomarkers of acute exacerbation. To the best of our knowledge, there is no study in the literature that evaluates the roles of both MPV and NLR in predicting acute exacerbation in patients with $\mathrm{BE}$.

Mean platelet volume is correlated with platelet function and activation [6]. Platelet activation that occurs in the process of inflammation can be measured indirectly through MPV. Mean platelet volume alone represents both platelet stimulation and the rate of platelet production [11]. CD62, CD63, GP IIB/IIIA, PF4, and thromboglobulin can be used as markers of platelet activation [12]. These tests are not routinely used due to their high cost and need of specialised equipment [13]. Measurement of mean platelet volume is a cheap, effective, and an easy method that is closely correlated with platelet function and activation and is used in the assessment of platelet function.

Recent studies show that platelets, one of the most important elements of the haemostasis process, also play a role in the development of immune response [14]. Usually in adult studies, it has been shown that there is platelet activation in different inflammatory lung diseases [8, 9, 15-21]. In the study by Bansal et al. [15] involving 100 patients with chronic obstructive pulmonary disease (COPD) and 100 healthy subjects, the MPV values in COPD patients were detected as being higher than those in the control group. Erden et al. [16] found MPV values to be higher during flare-up periods in their work about exacerbation

Table 2. Correlation of neutrophil count and C-reactive protein levels and correlation of mean platelet volume and neutrophil-to-lymphocyte ratio in patient groups during acute exacerbation and non-exacerbation chronic disease periods

\begin{tabular}{|c|c|c|c|c|}
\hline \multirow[t]{2}{*}{ Factor } & \multicolumn{2}{|c|}{ MPV (fl) } & \multicolumn{2}{|c|}{ NLR } \\
\hline & $r$ & $p$ & $r$ & $p$ \\
\hline \multicolumn{5}{|c|}{ The period of acute exacerbation } \\
\hline $\mathrm{CRP}(\mathrm{mg} / \mathrm{l})$ & 0.01 & 0.947 & 0.498 & $<0.001$ \\
\hline $\mathrm{WBC}\left(\times 10^{3} / 1\right)$ & -0.033 & 0.704 & 0.663 & $<0.001$ \\
\hline \multicolumn{5}{|c|}{ The period of chronic illness } \\
\hline CRP (mg/l) & 0.041 & 0.777 & 0.033 & 0.819 \\
\hline WBC $\left(\times 10^{3} / 1\right)$ & -0.029 & 0.841 & 0.441 & 0.001 \\
\hline
\end{tabular}


of COPD. In another study with 85 COPD patients, MPV values and the number of leukocytes were found to be significantly higher in COPD patients compared to the control group [17]. In contrast, in the study of Uysal et al. [18] evaluating children with BE, MPV levels in the acute exacerbation period were found to be significantly lower when compared to the non-exacerbation period and the control group. In asthmatic children, as another chronic airway disease, Tuncel et al. [19] did not find any difference between MPV levels of acute episodes and symptom-free periods. Similarly, in our study about children with asthma, there were no difference between MPV values in acute episodes and symptom-free periods [20]. As seen in the literature, different conclusions concerning use of MPV as a marker of inflammation are reported. In our study, no statistical difference was detected between MPV levels of acute exacerbation periods and non-exacerbation periods, so it can be concluded that MPV cannot be used as a marker for exacerbation. Different results in various studies may result from methodological changes. Also, MPV levels may be influenced by the method of blood sampling, care of blood samples, time between sampling and analysing, and the drugs used by the cases [18]. In our study, platelet counts in the acute exacerbation and non-exacerbation periods were significantly higher than in the control group. This result leads to the idea that increased platelet count is associated with chronic inflammation in BE, but measurement of MPV is not adequate for showing inflammation via platelets in cases of acute exacerbation.

NLR has been used as a marker for inflammation in several diseases because the physiological responses of circulating leucocytes in the human body to stress are an increase in the number of neutrophils and a decrease in the number of lymphocytes [21, 22]. In various studies about NLR, many domains such as systemic oxidative stress, activation of circulating inflammatory cells, an increase in plasma cytokines and acute phase proteins, weight loss, and skeletal muscle dysfunction are observed related to the inflammatory response in the lungs [9, 23-28]. Related to this, in studies of adult patients with COPD, significant rises in serum CRP levels and leukocyte counts in the period of acute exacerbation of COPD have been shown [23-25]. Drews et al. [26] showed an increase in sputum eosinophil counts in the majority of children with atopic asthma, and in contrast, a significantly higher sputum neutrophil rate in nonatopic children with asthma. Zhang et al. [27], in a study evaluating the blood cell counts and the percentages of neutrophils and eosinophils in sputum cytology in asthmatic patients, detected an increase of NLR in neutrophilic asthma and reported this finding to be a practical parameter especially in uncontrolled asthma. Furukawa et al. [28] showed that the number of neutrophils in sputum were increased in asthmatic children, and there is a small correlation between the NLR of the patients with the number of hospital admissions. In a study of 50 adult patients with asthma, Fu et al. [29] found that the number of neutrophils in the sputum is higher in asthmatic patients with systemic inflammation. Gunay et al. [30], in a study on 269 patients with COPD, showed that NLR were higher than controls in patients with stable COPD, and there is a positive relationship between CRP and NLR. Similarly, in our study, leukocyte count, absolute neutrophil count, NLR, and CRP values in children with $\mathrm{BE}$ were higher when compared to the control group. Also, a moderate positive correlation between NLR and CRP, with leukocyte count, was detected. Therefore, we think that NLR could be used as a marker to show acute exacerbation.

The limitations of our study are the insufficient number of patients and its retrospective nature. Thus, the effects of respiratory function tests in acute exacerbations and non-exacerbation periods, sputum cytology, colonisation status on NRL, and MPV could not be studied. We think that correlation analysis with proinflammatory cytokines, which has been shown to play a role in the inflammation process of acute exacerbation and progressive period of $\mathrm{BE}$ patients, such as IL-6, IL-8, TNF- $\alpha$, and ICAM, should be done in subsequent studies.

As a result of this study, it can be concluded that there may be a role played by platelets in the pathophysiology of BE, but MPV measurement is not a potential biomarker of acute exacerbations. In contrast, NLR and absolute neutrophil count, as a quick, cheap, and easily measurable feature, can be used as a marker for acute exacerbations of $\mathrm{BE}$ in children.

The authors declare no conflict of interest.

\section{References}

1. Re Redding GJ (2009): Bronchiectasis in children. Pediatr Clin North Am 56: 157-171.

2. Karadag B, Karakoc F, Ersu R, et al. (2005): Non-cystic fibrosis bronchiectasis in children: a persisting problem in developing countries. Respiration 72: 233-238.

3. Wilson CB, Jones PW, O'Leary CJ, et al. (1997): Validation of the St. George's respiratory questionnaire in bronchiectasis. Am J Respir Crit Care Med 156: 536-541.

4. Bloemen K, Van Den Heuvel R, Govarts E, et al. (2011): A new approach to study exhaled proteins as potential biomarkers for asthma. Clin Exp Allergy 41: 346-356.

5. van de Kant KD, Jansen MA, Klaassen EM, et al. (2012): Elevated inflammatory markers at preschool age precedepersistent wheezing at school age. Pediatr Allergy Immunol 23: 259-264.

6. Wiwanitkit V (2004): Plateletcrit, mean platelet volume, platelet distribution width: its expected values and correlation with parallel red blood cell parameters. Clin Appl Thromb Hemost 10: 175-178.

7. Kowal-Bielecka O, Kowal K, Lewszuk A, et al. (2005): B-thromboglobulin and platelet factor in bronchoalveolar lavage fluid of patients with systemic sclerosis. Ann Rheum Dis 64: 484-486. 
8. Yamamoto H, Nagata M, Tabe K, et al. (1993): The evidence of platelet activation in bronchial asthma. J Allergy Clin Immunol 91: 79-87.

9. Agustí AG, Noguera A, Sauleda J, et al. (2003): Systemic effects of chronic obstructive pulmonary disease. Eur Respir J 21: $347-360$

10. Chang AB, Reddind GJ (2006): Bronchiectasis. In: Chernick V, Boat T, Wilmott RW, Bush A, Kendig EL, editors. Kendig's disorders of respiratory tract in children. Philadelphia: WB Saunders Company; 463-476.

11. Khandekar MM, Khurana AS, Deshmukh SD, et al. (2006): Platelet volume indices in patients with coronary artery disease and acute myocardial infarction: an Indian scenario. J Clin Pathol 59: 146-149.

12. Tsiara S, Elisaf M, Jagroop IA, Mikhailidis DP (2003): Trombosits as predictors of vascular risk: Is there a practical index of trombosit activity? Clin Appl Thromb Hemost 9: 177-190.

13. Bath PM, Butterworth RJ (1996): Platelet size: Measurement, physiology and vascular disease. Blood Coagul Fibrinolysis 7: 157-161.

14. Idzko M, Pitchford S, Page C (2015): Role of platelets in allergic airway inflammation. J Allergy Clin Immunol 135: 1416-1423.

15. Bansal R, Gupta HL, Goel A (2002): Association of Increased Platelet Volume In Patients of Chronic Obstructive Pulmonary Disease: Clinical Implications. JIACM 40: 104-107.

16. Erden ES, Dokuyucu R, Demirköse M, et al. (2013): Assessment of mean platelet volume in chronic obstructive pulmonary disease during stable period and acute exacerbation. JCEI 4: 483-487.

17. Steiropoulos P, Papanas N, Nena E, et al. (2013): Mean platelet volume and platelet distribution width in patients with chronic obstructive pulmonary disease: the role of comorbidities. Angiology 64: 535-539.

18. Uysal P, Tuncel T, Erge D, et al. (2014): Does Mean Platelet Volume in Children with Bronchiectasis Predict Exacerbations? Int J Hematol Oncol 24: 54-59.

19. Tuncel T, Uysal P, Hocaoglu AB, et al. (2012): Change of mean platelet volume values in asthmatic children as an inflammatory marker. Allergol Immunopathol (Madr) 40: 104107.

20. Nacaroǧlu HT, İşgüder R, Bahceci SE, et al. (2016): Can mean platelet volume be used as a biomarker for asthma? Postepy Dermatol Alergol 33: 182-187.

21. Bhat T, Teli S, Rijal J, et al. (2013): Neutrophil to lymphocyte ratio and cardiovascular diseases: a review. Expert Rev Cardiovasc Ther 11: 55-59.

22. Zahorec R. (2001): Ratio of neutrophil to lymphocyte counts - Rapid and simple parameter of systemic inflammation and stress in critically ill. Bratisl Lek Listy 102: 5-14.

23. Rohde G, Borg I, Wiethege A, et al. (2008): Inflammatory response in acute viral exacerbations of COPD. Infection 36: 427-433.

24. Ulasli SS, Ozyurek BA, Yilmaz EB, Ulubay G. (2012): Mean platelet volume as an inflammatory marker in acute exacerbation of chronic obstructive pulmonary disease. Pol Arch Med Wewn 122: 284-290.

25. Saldias PF, Diaz PO, Dreyse DJ, et al. (2012): Etiology and biomarkers of systemic inflammation in mild to moderate COPD exacerbations. Rev Med Chil 140: 10-18.

26. Drews AC, Pizzichini MM, Pizzichini E, et al. (2009): Neutrophilic airway inflammation is a main feature of induced sputum in nonatopic asthmatic children. Allergy 64: 15971601.

27. Zhang XY, Simpson JL, Powell H, et al. (2014): Full blood count parameters for the detection of asthma inflammatory phenotypes. Clin Exp Allergy 44: 1137-1145.

28. Furukawa T, Sakagami T, Koya T, et al. (2015): Characteristics of eosinophilic and non-eosinophilic asthma during treatment with inhaled corticosteroids. J Asthma 52: 417-422.

29. Fu JJ, Baines KJ, Wood LG, Gibson PG (2013): Systemic inflammation is associated with differential gene expression and airway neutrophilia in asthma. OMICS 17: 187-199.

30. Günay E, Sarınç Ulaşlı S, Akar O, et al. (2014): Neutrophil-to-lymphocyte ratio in chronic obstructive pulmonary disease: a retrospective study. Inflammation 37: 374-380. 